\title{
Short and long period variable stars in the Carina dwarf Spheroidal galaxy
}

\author{
M. Monelli ${ }^{1}$, A.R. Walker ${ }^{2}$, G. Bono ${ }^{1}$, R. Buonanno ${ }^{1}$, F. Caputo $^{1}$, \\ M. Castellani ${ }^{1}$, V. Castellani ${ }^{1}$, C.E. Corsi ${ }^{1}$, M. Dall'Ora ${ }^{1}$, L. Pulone ${ }^{1}$,

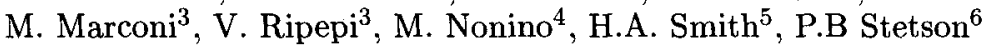 \\ ${ }^{1}$ INAF Osservatorio Astronomico di Roma, via Frascati 33, 00040 \\ Monteporzio Catone, Roma, Italy \\ ${ }^{2}$ Cerro Tololo Inter-American Observatory, National Optical Astronomy \\ Observatories, Casilla 603, La Serena, Chile \\ ${ }^{3}$ INAF Osservatorio Astronomico di Capodimonte, via Moiariello 16 \\ 80131, Napoli, Italy \\ ${ }^{4}$ INAF Osservatorio Astronomico di Trieste, via Tiepolo 11, 43131 \\ Trieste, Italy \\ ${ }^{5}$ Department of Physics and Astronomy, Michigan State University, \\ East Lansing, MI 48824, USA \\ ${ }^{6}$ Dominion Astrophysical Observatory, Herzberg Institute of \\ Astrophysics, NRC, 5071 West Saanich Road, Victoria, BC V9E 2E7, \\ Canada
}

\begin{abstract}
We present first results concerning the detection of variable stars in the Carina dwarf Spheroidal from $B, V$ images collected with the 4-m CTIO telescope. We show a sample of candidate variables spanning from the tip of the Red Giant Branch down to the Main Sequence turn off. Finally, we discuss the future photometric and spectroscopic developments of this project.
\end{abstract}

\section{Introduction}

Dwarf spheroidal galaxies ( $\mathrm{dSph}$ ) are considered important and interesting astrophysical objects. It is widely thought that they played a key role in galaxy formation, being the first and smallest structures that appeared in the universe. Current $\Lambda$ Cold Dark Matter (CDM) theories predict that these structures are the primordial building blocks that formed bigger galaxies. During the last few years many efforts, both on the theoretical as well as on the observational side, have been focused on the small relics of this accretion process. The Local Group (LG) offers the unique possibility to resolve the stars in these loose, faint, low density galaxies and study in detail their star formation history (SFH), chemical evolution and dynamical properties. We started a long-term project on the Carina $\mathrm{dSph}$, aimed at studying the properties of both constant and variable stars. Carina dSph is the prototype of a complex SFH, which proceeded via subsequent bursts of different intensity and duration. This means that we can 
study simultaneously old $(\simeq 12 \mathrm{Gyr})$ and very young ( $\leq 1 \mathrm{Gyr})$ populations, and that we can find variable stars in different evolutionary phases.

\section{Data sets}

DSph galaxies are typically uncrowded systems but their photometric study is complicated by their small central density and low surface brightness. Moreover, the nearby LG dSphs cover a huge area in the sky (of the order of square degrees): for a long time this has strongly limited the sample of stars detected with standard CCDs. Things have drastically changed with the wide field cameras $\left(\simeq 30^{\prime} \times 30^{\prime}\right)$ which give the opportunity to sample a large fraction of the stellar content of these objects. The Carina $\mathrm{dSph}$ has a tidal radius of $\simeq 29^{\prime}$ (Mateo 1998), and we studied its central region with two different wide-field cameras: the WFI mounted on the 2.2-m ESO and the Mosaic II detector on the 4-m CTIO telescope. We present the first results based on this latter data set. Data were obtained on three non-consecutive nights between 1999 December and 2000 January; $54 B, V$ pairs were obtained, for a total integration time of $13200 \mathrm{~s}$ and $21000 \mathrm{~s}$ in the $V$ - and $B$-bands respectively.

Observations were optimized for the detection of short period variables, from RR Lyrae stars down to the Main Sequence Turn Off (MSTO) stars. Standard IRAF (Valdes 1997) routines were applied for bias subtraction and flat field correction. Photometric reduction was performed with the DAOPHOTII/ ALLSTAR/ALLFRAME package (Stetson 1987, 1994). Particular attention was paid to the selection of stars used to model the PSF. To ensure an adequate sampling of the spatial variation of the PSF along the frame, we selected $\simeq 150$ stars spread over the entire field of view of each chip. We used the WelchStetson (Welch \& Stetson 1993) index to select candidate variable stars. This index basically estimates the scatter of measurements around the mean magnitude. Since luminosity variations are correlated in different bands, the strength of this method is that it looks for correlated deviations from the mean in two colors. We coupled subsequent measurements in the $B$ - and $V$-bands, estimating the scatter from the mean. If a star is constant, the difference between the measures and the mean is expected to be small and randomly distributed around zero. If a star shows systematic variations in its luminosity, the scatter is greater and the index can be significantly greater than zero.

We present candidate variable stars from two central chips of the 4-m CTIO data set. Fig. 1 shows the color-magnitude diagram from the two selected chips with the candidate variable stars superimposed. This sample was cleaned by using the sharpness parameter. Many interesting features show up from this diagram and deserve more accurate analysis. We have candidate variable stars ranging from the tip of the RGB down to the MSTO of the intermediate-age population. RR Lyrae stars and Anomalous Cepheids (see Dall'Ora et al. 2003), that is, old and intermediate-age stars undergoing He-core burning, populate the Cepheid instability strip. At lower luminosities, in the region of the MSTO, we find evidence of the presence of $\delta$ Scuti stars (Mateo et al. 1998). The detection of variable stars in this region has many interesting implications. Owing to the complex SFH of this galaxy, in this region of the color-magnitude diagram $\delta$ Scuti stars of the intermediate age population that are now leaving the main sequence 


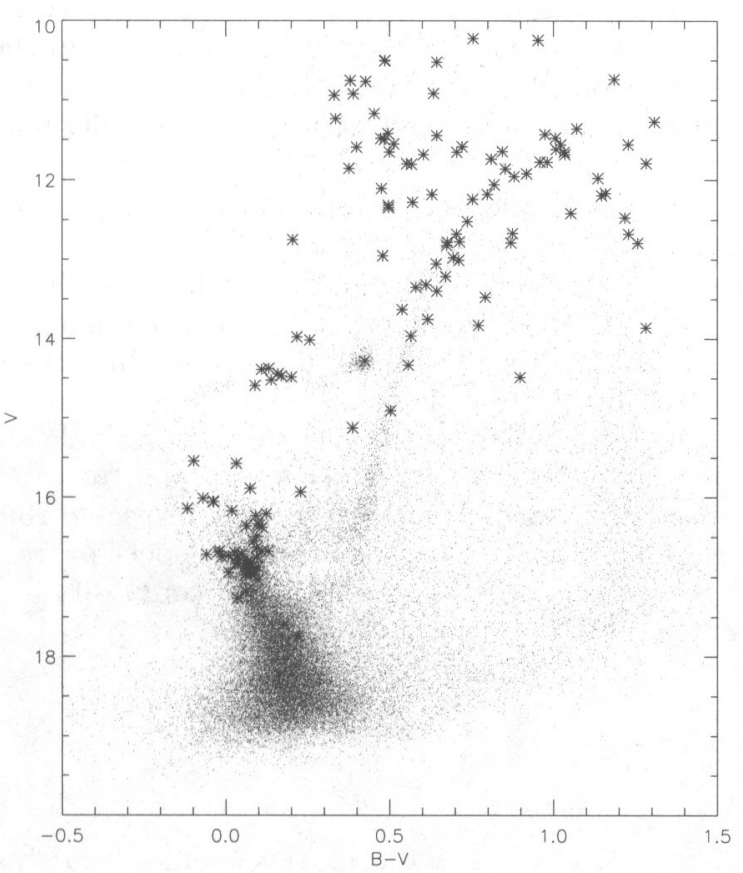

Figure 1. Color-magnitude diagram of the two selected chips. We superimpose our candidate variables selected according their sharpness parameters: $\mid$ sharp $\mid \leq 0.05$. Note that the candidate field variables are well below the saturation threshold.

$\left(\mathrm{M} \simeq 1.2 \mathrm{M}_{\odot}\right)$ coexist with oscillating Blue Stragglers of the old population. This offers an unprecedented opportunity to study in the same system these two types of variables and shed light on their origin and pulsational properties.

We also note the presence of candidate variable stars along the RGB and the AGB: these are presumably long-period variables (Wood 2000; Ita et al. 2002; Kiss \& Bedding 2003). Moreover, we found several interesting candidates among field stars.

\section{Future developments}

We collected multi-band and multi-epoch data of the central region of Carina dSph with two wide-field cameras. The first effort we made to improve our data set was an extensive study of all data available in literature. We ended up with a huge data base of images:

- $\simeq 70 \mathrm{~B}, I$ images collected with the 1.5-m CTIO telescope in 1992 (SmeckerHane, Stetson);

$-\simeq 150 \mathrm{~V}+70 \mathrm{I}$ images collected with the 1.5-m CTIO telescope in 1996 (Kuhn); 
- $\simeq 70 \mathrm{~V}+40 \mathrm{I}$ images from the OGLE I database 1998 (Udalski);

$\bullet \simeq 7 V, I$ images collected with the 4-m CTIO telescope in 2000 (Gallart);

$-\simeq 48$ BVI images from the EIS project in 2001;

$-\simeq 120 \mathrm{~V}+30 B I$ images collected with the 2.2-m ESO telescope from 1999 to 2001 ;

- Strömgren $u, v, b, y$ and Ca images collected in 2001 (Bono);

- $\simeq 30 \mathrm{BV}$ images collected in 2002 (Bono);

- and some spare images from HST and VLT archives in the $V, I$ bands.

Work is in progress, and all different sub-sets have been independently analyzed. The main goal of this project is to simultaneously reduce the entire photometric data set with ALLFRAME. This will supply homogeneous reduction and calibration, and in turn the opportunity to detect variable stars with periods ranging from few hours to years. Moreover, we plan to extend this work by collecting mid-resolution spectra with VIMOS: we selected roughly 500 targets among RGB, red clump, and HB stars. The new spectroscopic data will allow us to estimate the radial velocities and chemical composition of a large sample of stars distributed over the main body of the galaxy.

\section{Conclusions}

We presented photometric $B, V$ data for the Carina dwarf Spheroidal Galaxy collected with the Mosaic II camera. We showed preliminary results concerning the detection of variable stars in the central region of this galaxy. We have bona fide candidates from the tip of the AGB/RGB down to the MSTO. We have gathered many data sets available in the literature, and we are in the process of reducing them all at once, covering a time baseline of almost $10 \mathrm{yr}$. This will allow us to study both short and long period variable stars in Carina, and hopefully disentangle different groups of variables in the region of the MSTO.

\section{References}

Dall'Ora, M., et al. 2003 AJ, 126, 197

Ita, Y., et al., 2002, MNRAS, 337, 31

Kiss, L.L, Bedding, T., 2003, MNRAS, 343, 79

Mateo, M., 1998, ARA\&A, 36, 435

Mateo, M., Hurley-Keller, D., Nemec, J., 1998, AJ, 115, 1856

Stetson, P.B., 1987, PASP, 99, 191

Stetson, P.B., 1994, PASP, 106, 250

Valdes, F. 1997, in ASP Conf. Ser., Vol. 125, Astronomical Data Analysis Software and Systems VI, eds G. Hunt \& H.E. Payne, (San Francisco: ASP), 455

Welch, D.L., Stetson, P.B., 1993, AJ, 105, 1813

Wood, P.R. 2000, PASA, 17, 18 


\section{Discussion}

Clementini: How did you make the detection of variable stars in your frames?

Monelli: We used a modified Welch-Stetson index.

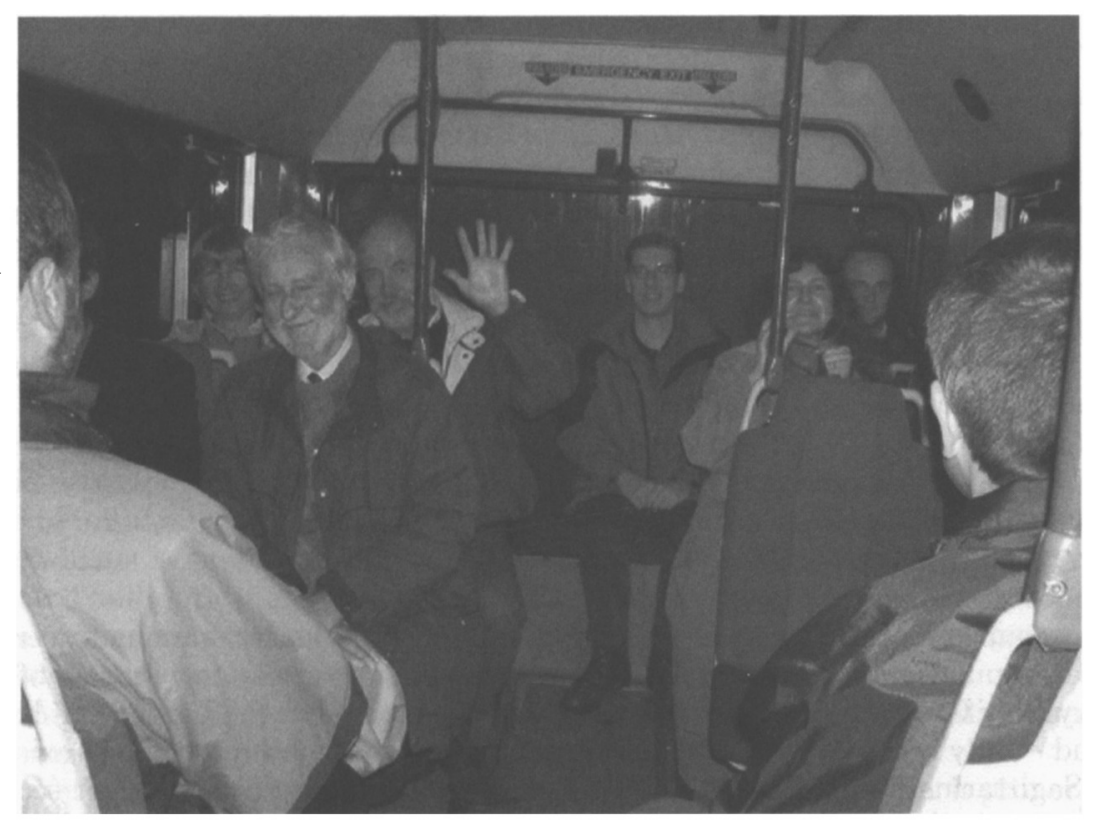

Michael Feast, Anna and Sławek Rucinski, Joris de Ridder, Joyce Guzik and Marc-Antoine Dupret on the bus to the banquet. 